\title{
Clinical governance: costs and benefits
}

\section{Kieran Walsh ${ }^{*}$}

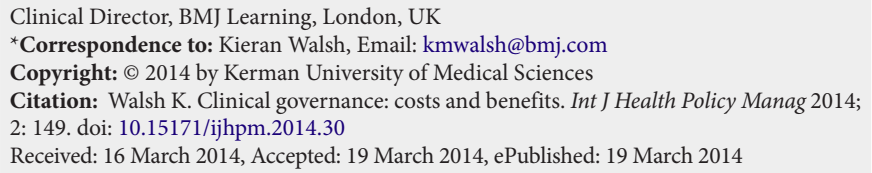

Dear Editor,

Ravaghi et al. should be congratulated for offering a fascinating insight into the views of senior managers on the implementation of clinical governance (1). Clearly many had experience of challenges in implementation and there were different types of challenges. However, a common theme ran through many of the challenges, and that theme is one of cost. The managers did not mention cost explicitly but the issue was clearly implicit. They spoke of the need for more resources, support and staff engagement-all of which are associated with costs. We would do well to ask outright: what are the costs of implementing clinical governance? Such costs are likely to be significant, and are likely to be made up of all the components of clinical governance including, as the authors outline, "clinical effectiveness, clinical audit, risk management, patient and public involvement, education and training, staff management, and use of information" (2).

Each of these is expensive-not least education and training of staff. To justify such costs, healthcare systems need to see real outcomes from clinical governance in terms of improved quality of care for patients and the public. Perhaps this perspective should make us re-focus our clinical governance efforts upon clinical quality improvement and cost efficiency. Clinical quality improvement may in fact go hand in hand with cost efficiency. If individual healthcare professionals and teams concentrate their efforts on areas where institutions need to improve then that would be a good start. They could then measure quality at baseline, put measures in place to improve quality and continually re-measure. In the medium to long-term quality improvement processes should be embedded. If done properly, quality improvement should save costs. It should cut out inefficient practices and prevent expensive medical errors. Individuals and teams should share their learning throughout institutions and ensure that mistakes are not repeated and that in effect organisations as a whole learn.

If we do not take this approach, clinical governance will likely become an ever ballooning cost. If we do, clinical governance will develop focus, and with such focus, will come tangible benefits through quality improvement and cost saving.

\section{Ethical issues}

Not applicable.

\section{Competing interests}

The author declares that he has no competing interests.

Author's contribution

$\mathrm{KW}$ is the single author of the manuscript.

\section{References}

1. Ravaghi H, Heidarpour P, Mohseni M, Rafiei S. Senior managers' viewpoints toward challenges of implementing clinical governance: a national study in Iran. Int J Health Policy Manag 2013; 1: 295-9.

2. Jafari $\mathrm{GH}$, Khalifeh Gari S, Danaie Kh. Hospital Accreditation Standards in Iran. Tehran: Ministry of Health and Medical Education; 2011. p. 1-3. 